\title{
Comparison of the Past Two Solar Minima from the Perspective of the Interior Dynamics and Dynamo of the Sun
}

\author{
Mausumi Dikpati
}

Received: 30 September 2010 / Accepted: 23 May 2011 / Published online: 17 June 2011

(C) The Author(s) 2011. This article is published with open access at Springerlink.com

\begin{abstract}
Solar cycle 23 behaved differently than cycle 22 in many ways. Certain properties, namely the long minimum at the end of cycle 23, weakening of polar fields, shrinking of polar coronal holes, reduction in the terrestrial atmospheric neutral density layer, have been identified as unusual compared to several past cycles. The origin of these differences most likely lies in the ways the dynamo has operated that led to distinctly different generation and evolution of the large-scale magnetic fields in cycles 22 and 23. Certain differences in the properties of Galactic-Cosmic Rays during cycles 22 and 23 have recently been explained by the differences in evolutionary pattern of coronal holes, which are linked to the dynamogenerated large-scale magnetic fields. In this paper, I will discuss the differences in the solar interior dynamics, particularly the properties of flow fields and their influence in governing the evolution of dynamo-generated magnetic fields during cycles 22 and 23, respectively.
\end{abstract}

Keywords Solar activity $\cdot$ Dynamo $\cdot$ Meridional circulation

\section{Introduction}

The observational records of sunspot data indicate that the minimum between cycles 22 and 23 has been more than twice as long as that at the end of cycle 22 . The monthly smoothed sunspot number (ssn) has been plotted in Fig. 1. If we define a solar minima in ssn for a value of ssn $<15$, then it yields 9 and 38 months for the lengths of minima at the end of cycles 22 and 23, respectively. There is no universal definition for the cut-off value of ssn for minimum conditions. However, no matter what cut-off value is being considered for determining the length of minimum, the essence is - the minimum at the end of cycle 23 was much longer than that at the end of cycle 22. In fact, the minimum at the end of cycle 23 has been unusually long, the longest in 100 years. One has to go back nine cycles in order to find such a long minimum. It has also been a deep minimum; the lowest ssn was 1.7, whereas the lowest ssn at the end of cycle 22 was 12.6.

M. Dikpati (凶)

HAO/NCAR, 3080 Center Green, Boulder, CO 80301, USA

e-mail: dikpati@hao.ucar.edu 
Fig. 1 Monthly smoothed sunspot number data from Royal Observatory of Belgium (www.sidc.be) are plotted in blue filled curve for a time span from January, 1986 to February, 2010. Lengths of minima at the end of cycles 22 and 23 are indicated by thick yellow lines

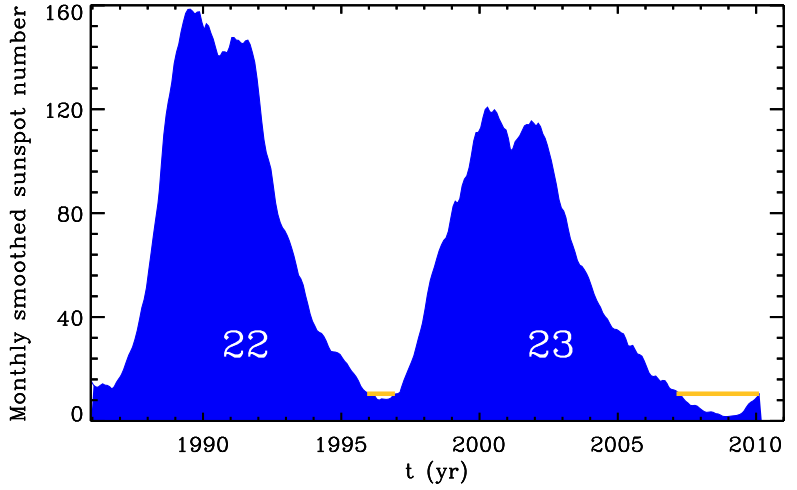

The effect of this unusually long, deep minimum has been observed in various other solar cycle indices, such as in f10.7 radio flux, total solar irradiance (TSI) (Fröhlich 2009; Domingo et al. 2009), atmospheric neutral density (Solomon et al. 2010), as well as in the trend of solar p-mode frequency (Tripathy et al. 2010) and torsional oscillation (Howe et al. 2009). These unusual features observed in cycle 23 are most likely due to the dynamo operating in a somewhat different fashion compared to the previous cycles. In this context, we can mention other relevant observations by the Ulysses spacecraft in the modulation of the galactic cosmic rays-in cycle 22 the decrease in the component of the recurrent cosmic rays arising from the fast solar wind showed a clear maximum from $25^{\circ}$ to $40^{\circ}$ and beyond, whereas in cycle 23 there was no such significant decrease (Dunzlaff et al. 2008). As an explanation the authors have suggested that the absence of large stable coronal hole structures during cycle 23 might have caused this difference. Since the large-scale coronal structure is most likely governed by the dynamo-generated cyclic magnetic fields, it is important to understand the causes that created the differences in the operation of the Sun's global dynamo during cycle 23 compared to that in cycle 22 .

These observations and associated physical reasonings motivate us to seek answers to several questions-whether such a long, deep minimum at the end of cycle 23 would lead to a strong or weak cycle 24; why cycle 23 lasted so long, for about 12.5 years compared to past four cycles (19 to 22) which had an average duration of 10.5 years; why the large stable coronal holes disappeared in cycle 23.

Another important feature of the last minimum is an unusually low value of the polar fields, a fraction of $50 \%$ of that during the minimum of cycle 22. If polar fields originate from the Babcock-Leighton type decay of tilted, bipolar active regions (Babcock and Babcock 1955; Leighton 1969), they will have to be the follower of a cycle. It is an observational fact that the relative amplitude of cycle 23 was $20 \%$ smaller than that of cycle 22 . If at the end of a given cycle the polar field has one unit, in the next cycle it takes two units of polar fields coming from the surface poloidal source to reverse the remnant polar field and have the new polar fields reach minus one unit. But if the surface poloidal source is $20 \%$ smaller in the new cycle than the previous one (as cycle 23 was compared to cycle 22) there are only 1.6 units of new, negative, polar fields available that can be used to reverse the old, positive, polar field and establish the new, negative, polar field. This means the new, negative, polar field will be only 0.6 units, or $40 \%$ smaller in relative amplitude than that of the previous cycle. However, we do not attempt a detailed discussion of polar fields in this paper, because that will be discussed in details in another paper by Jiang et al. (2011, this issue). An extensive review of this topic has recently been published by Sheeley (2010). 


\section{Can the Length of a Minimum be Used to Predict the Next Cycle's Strength?}

There exists a prevailing view in the community that a long minimum leads to a weak following cycle, for example, see Hathaway (2010). With our present knowledge of how a dynamo operates inside the Sun and produces a cyclic evolution of the Sun's global magnetic fields, it is not possible to find any physical reason behind this notion. So let us investigate whether we have any statistical evidence to hold the concept that a weak cycle will follow after a long minimum.

Dikpati et al. (2010a, 2010b) have recently estimated the correlation coefficients between the length of a cycle minimum and the following peak minus preceding peak. Using a cutoff value of 15 for the monthly ssn (downloaded from the website of Royal Observatory of Belgium, www.sidc.be) and using a cutoff value of 180 for the spot area data averaged over 13 rotations as previously used by Dikpati et al. (2006), they estimated the lengths of minima for 23 ssn cycles and 12 spot-area cycles and examined whether they can say something about the following $n$th cycle's amplitude relative to the preceding $(n-1)$ th one's, based on the length of the minimum between the $n$th and $(n-1)$ th cycles. They correlated the length of minimum with the difference in amplitude between the following and preceding cycles so that any longer term trends, regardless of origin, get eliminated. This correlation was found to be insignificant-with correlation coefficients of $r=-0.13$ for monthly ssn and $r=-0.10$ for spot-area data (see Fig. 2a), with a relative chance of about $70 \%$ that these correlations are random. This means that it is not possible to predict whether the next cycle will be stronger or weaker than the previous one when we use the length of minimum as the predictor. Therefore the current long minimum at the end of cycle 23 does not necessarily portend a weaker cycle 24 than 23 .

By defining the depth of a minimum as the least spot number or the least spot area within the span of a cycle minimum, Dikpati et al. (2010a, 2010b) also examined whether there is any correlation between the length of a minimum and its depth. They found a strong anticorrelation between the length and depth of a minimum, the correlation coefficient $r$ being -0.75 for spot number, and -0.79 for spot area, with a small probability that either of these values is due to chance is $(1-2) \times 10^{-3}$. Figure $2 \mathrm{~b}$ shows a scatterplot with linear fits to both data sets.

Why a long minimum is also a deep minimum can be physically explained using our general concept of dynamo processes. During the minimum phase of a solar cycle, oppositely directed toroidal bands are in close proximity on the two sides of the equator (see Fig. 2c); in the case of a long minimum they both have more time to annihilate each other, leading to fewer or no eruptions of low-latitude spots.

\section{Why Did Cycle 23 Last so Long?}

In flux-transport dynamos the cycle length is primarily determined by the speed and extent of the meridional circulation (Dikpati and Charbonneau 1999). The speed of the meridional flow at the surface has been measured for many years by various instruments. Most models were built assuming a meridional circulation profile that peaks around mid-latitudes, see for example Küker et al. (2001), Bonanno et al. (2002), Guerrero and Muñoz (2004), Jouve and Brun (2007), Jouve et al. (2008), Hotta and Yokoyama (2010). With such a prescribed profile, numerical experiments with a flux-transport dynamo including a slow-down in the meridional flow speed, as observed during 1996-2003 (Haber et al. 2002; Basu and Antia 2003), produced a forecast of about 1.5 year delay in the onset of cycle 24 (Dikpati 2004), with a prediction of 12 years, but in reality it lasted about six months longer. 
Fig. 2 Scatter-plot for difference between following and preceding cycles' peaks as function of length of minima between following and preceding cycles (panel (a)); scatter-plot for length and depth of minima (panel (b)); and a schematic diagram showing a configuration of solar minimum toroidal bands as would be generated at the base of the convection zone by the shearing mechanism of a dynamo (panel (c)). The frames of this figure have been adapted from Figs. 1, 2 and 3 of Dikpati et al. (2010a, 2010b)

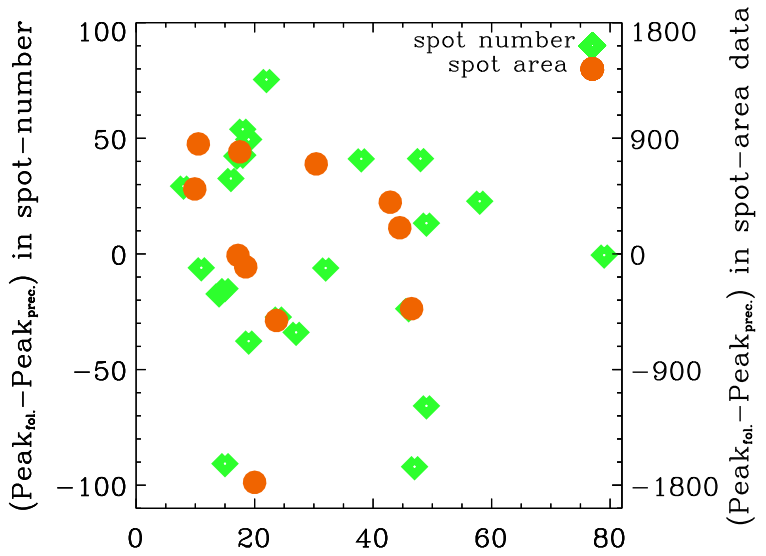

(a)

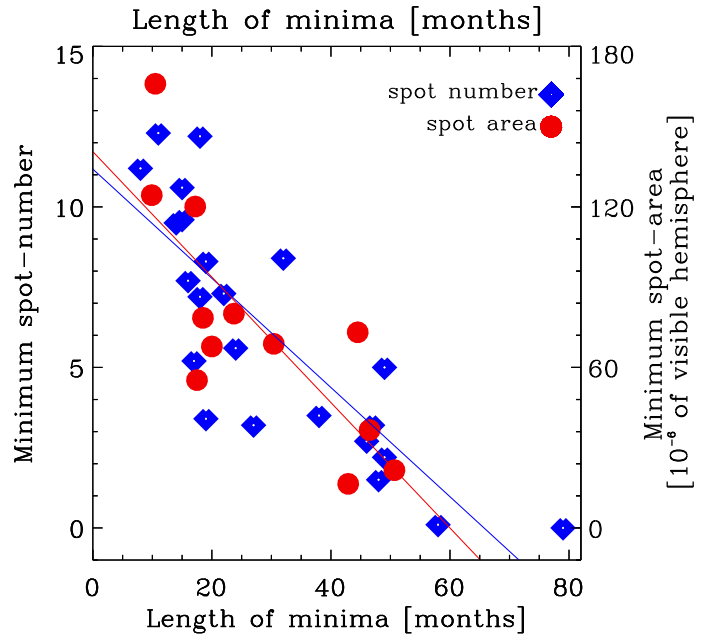

(b)

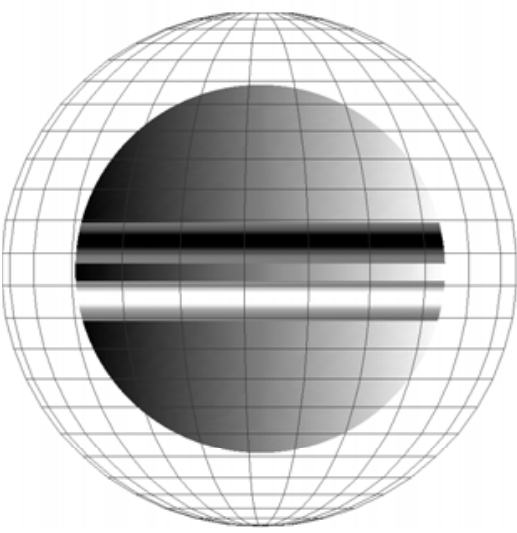

(c)

In order to investigate what caused the additional delay in the onset of cycle 24, Dikpati et al. (2010a, 2010b), Ulrich (2010) re-examined the behavior of the Sun's plasma flow, specifically how its properties changed during cycle 23 from that in cycle 22. Extending the analysis of long-term Mount Wilson Observatory (MWO) data previously performed in 


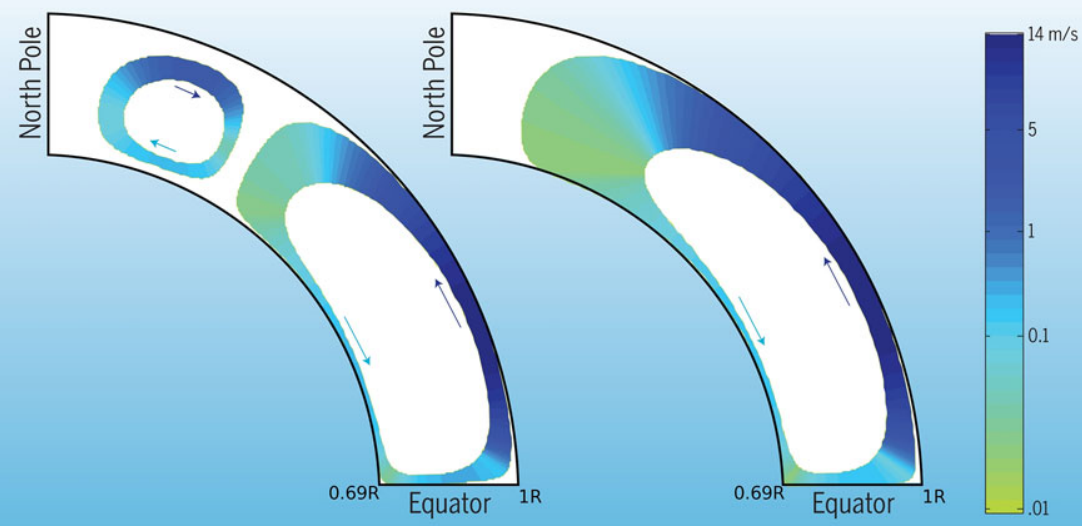

Fig. 3 Characteristics of the Sun's conveyor belts during cycles 22 (left frame) and 23 (middle frame) as obtained from surface observations combined with mass conservation. Note that the top and bottom boundaries of the flow are at the surface $(1 R)$ and at $R_{b}=0.69 R$. A colorbar at the right indicates the flow speeds presented in the left and middle frames

2005 by Ulrich and Boyden (2005), we found that the Sun's surface plasma was flowing poleward all the way up to the pole during the major part of cycle 23 , whereas during cycle 22 , the poleward surface flow ended around $60^{\circ}$ latitude, beyond which it became equatorward. Our analysis also indicates that the maximum average flow speed at the surface was not different during cycles 23 compared to that during cycle 22 .

Combining the above information from MWO observations of the poleward surface flow with the constraint of mass conservation, we can construct the equatorward return flow. The mathematical prescription of this flow is given by the following expression (see Dikpati and Choudhuri 1994) for the stream function $(\psi)$ :

$$
\psi r \sin \theta=\psi_{0}\left(\theta-\theta_{0}\right) \sin \left[\frac{\pi\left(r-R_{b}\right)}{\left(R-R_{b}\right)}\right]\left(1-\mathrm{e}^{-\beta_{1} r \theta^{\epsilon}}\right)\left(1-\mathrm{e}^{\beta_{2} r(\theta-\pi / 2)}\right) \mathrm{e}^{-\left(\left(r-r_{0}\right) / \Gamma\right)^{2}},
$$

in which, $R_{b}$ denotes the bottom boundary of the flow, $\beta_{1}$ and $\beta_{2}$ control the concentration of the streamlines at the polar and the equatorial regions respectively, $\epsilon$ with a proper choice of value $>2$ can avoid unphysical flow at the pole, combination of $r_{0}$ and $\Gamma$ determines at which depth the poleward flow becomes zero before returning towards the equator. The parameter $\theta_{0}$ determines the cell-size in latitude, namely $\theta_{0}=0$ means a single-cell flow going all the way to the pole. The values selected for these parameters are: $R_{b}=0.69 R, \beta_{1}{ }^{1}=0.1 /\left(1.09 \times 10^{10}\right) \mathrm{cm}^{-1}, \beta_{2}=0.3 /\left(1.09 \times 10^{10}\right) \mathrm{cm}^{-1}, \epsilon=2.00000001$, $r_{0}=\left(R-R_{b}\right) / 5, \Gamma=3 \times 1.09 \times 10^{10} \mathrm{~cm}$ and $\theta_{0}=0.14 \pi$ and 0 for the left and right frames of Fig. 3. This choice of the set of parameter values produce a flow pattern that peaks at $24^{\circ}$. As discussed in Dikpati et al. (2005), the dimensionless length in our calculation is

\footnotetext{
${ }^{1}$ The parameter values for $\beta_{1}, \beta_{2}$ and $\Gamma$ were given in dimensionless units in the GRL paper by Dikpati et al. (2010a, 2010b), whereas other parameters were given in dimensional units; we thank Dr. Luis Eduardo Antunes Vieira for helping us catching that.
} 
Fig. 4 A two-dimensional map of dynamo half-cycle period (corresponding to one sunspot cycle period), obtained from the simulations of a flux-transport dynamo, as function of latitude extent of primary meridional flow cell and maximum poleward surface-flow speed. Thick continuous white line denotes the contour of an 11-year sunspot cycle period. Thin white lines denote the contours of other periods in half-year intervals. The horizontal broken line marks the maximum surface-flow speed of $14 \mathrm{~m} / \mathrm{s}$. The positions of cycles 22 and 23 in this map are shown by semi-transparent gray patches [adapted from Dikpati et al. (2010a, 2010b)]

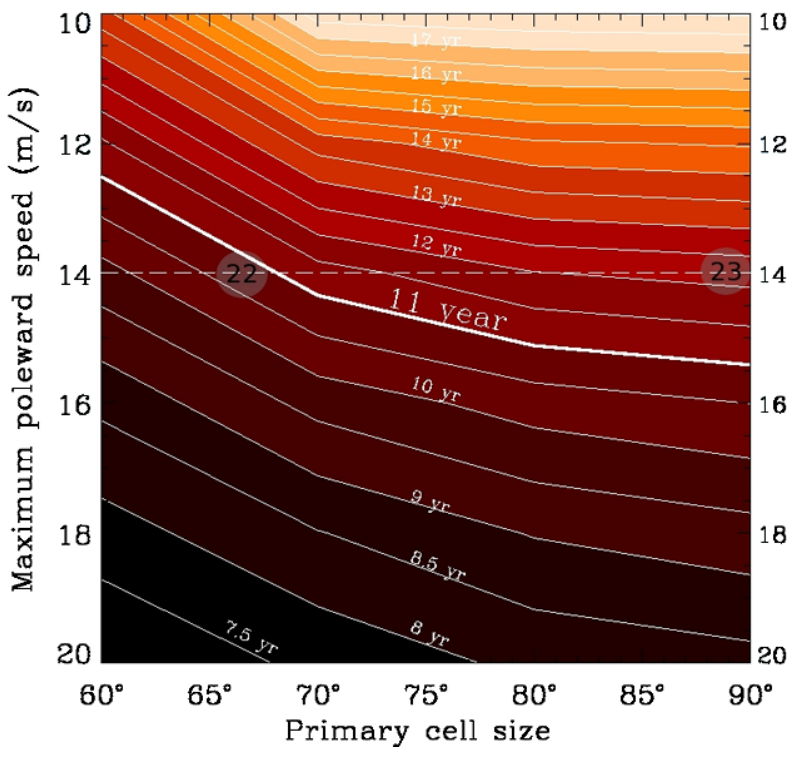

$1.09 \times 10^{10} \mathrm{~cm}$ and the dimensionless time is $1.1 \times 10^{8} \mathrm{~s}$, which respectively come from taking the inverse of the dynamo wavenumber, $k=9.2 \times 10^{-11} \mathrm{~cm}^{-1}$, as one unit of dimensionless length, and the inverse of the dynamo frequency, $v=9.1 \times 10^{-9} \mathrm{~s}^{-1}$, as one dimensionless time. If a full dynamo wavelength is defined as the full wavelength of a 22year magnetic cycle, then the migration of sunspots from about $40^{\circ}$ latitude to the equator represents a half wavelength of a dynamo cycle. A full dynamo wavelength in dimensional and nondimensional units are then $2 \pi \times 1.09 \times 10^{10} \mathrm{~cm}$ and $2 \pi$ respectively, and the mean dynamo cycle period in those units are 22 years and $2 \pi$. Thus, in dimensionless units, the above parameters are: $R_{b}=4.41, \beta_{1}=0.1, \beta_{2}=0.3, \epsilon=2.00000001, r_{0}=\left(R-R_{b}\right) / 5$, $\Gamma=3$ and $\theta_{0}=0$.

The characteristics of the Sun's conveyor belts during cycle 22 and 23, presented in Fig. 3, can be obtained by setting the values of $\theta_{0}$ respectively at $0.14 \pi$ and 0 . A longer conveyor belt in cycle 23 , associated with a slower return flow, compared to a shorter primary belt in cycle 22 , caused the cycle 23 to persist about two years longer than cycle 22 . Figure 4 shows the simulated cycle lengths as function of the extent of the Sun's primary conveyor belt and the maximum surface flow speed. As expected, simulations from a flux-transport dynamo produce the shortest cycles for faster flow speed and shorter primary belts-a parameter space that is located at the bottom-left corner of Fig. 3. Conversely the longest cycles are located at the top-right corner. As the average poleward surface flow speed did not differ much during cycles 22 and 23 , it is primarily the length of the conveyor belt that determined the cycle lengths in the cases of cycle 22 and 23 (see their positions marked in gray circles in Fig. 4). In fact, all past and future cycles can have a place on such a diagram if the surface flow speed and the latitudinal extent of the primary conveyor belt would be known from observations.

We know from sunspot-cycle observations that not just cycle 22 but the past four cycles, cycles 19 to 22 were short cycles with an average length of $\approx 10.5$ years. Analyzing MWO data from further back into the past Ulrich (1993) had already shown that a high-latitude reverse meridional flow cell indeed existed during cycles 20 and 21 . Thus, according to our flux-transport dynamo model, the flux must have been transported via a shorter path of the 
conveyor belts during those cycles, hence rendering an average duration of 10.5 years for cycles 20 and 21.

These observational facts leads to an obvious question-is a shorter primary flow cell ending around $60^{\circ}$ latitude and being associated with a reverse cell beyond $\approx 60^{\circ}$ latitude the Sun's more common choice? Dikpati and Gilman are currently developing a theory of meridional circulation at high-latitudes. Preliminary calculation shows that the Sun's preferred solution is to have a high-latitude reverse cell. Rather, the primary cell going all the way to the pole, as it did in cycle 23 , was unusual.

\section{Do We Know Why Large Stable Coronal Holes Disappeared in Cycle 23?}

Observations by the Ulysses spacecraft revealed that the recurrent cosmic rays measured in the fast solar wind showed distinct differences in cycles 22 and 23. A large, stable coronalhole structure was present during cycle 22, but not in cycle 23. The polar coronal-hole shrank during the declining phase of cycle 23 (Kirk et al. 2009) and a part of the coronal whole structure existed in the equatorial region (Abramenko et al. 2009).

The large-scale magnetic structure of the corona is most likely governed by the cyclic evolution of the dynamo-generated fields. We do not know the details of the interior dynamics that behaved differently during cycles 22 and 23, but we now know that the meridional circulation pattern behaved distinctly differently, namely a large flow cell persisted during cycle 23 going all the way to the pole from the equator at the surface, whereas during cycle 22 the flow pattern had two cells, with the primary cell going up to $\approx 60^{\circ}$ and then sinking down towards the bottom of the convection zone (cf., Fig. 3). Taking the dynamo-generated poloidal fields from Dikpati et al. (2010a, 2010b) and scaling the amplitudes accordingly to correspond to that of cycles 22 and 23, we use them as input to a simple 2D potential-fieldsource-surface (PFSS) model of the corona, and expand them up to $2.5 R_{\odot}$. This is a very
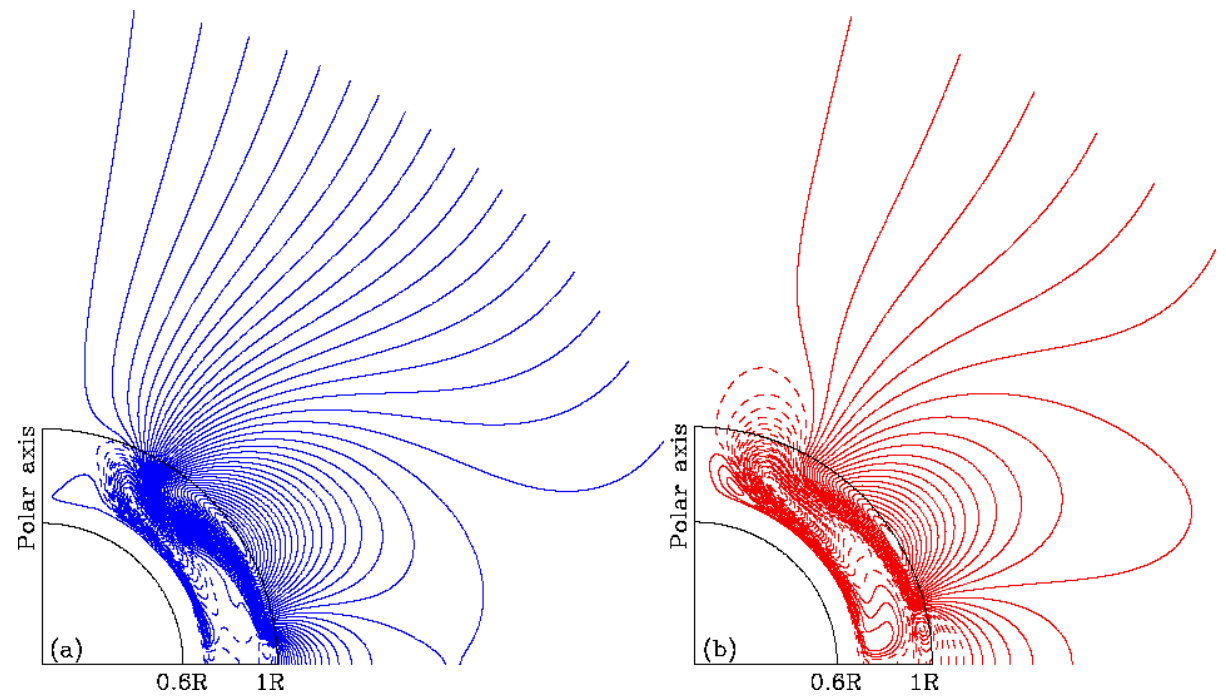

Fig. 5 Large-scale simulated corona derived from the PFSS extrapolation of a flux-transport dynamo model that ran with two meridional circulation patterns characteristics of cycle 22 and 23 as shown in Fig. 3; frames (a) and (b) respectively represent the corresponding coronal structures; open lines represent coronal-hole regions 
simplified 2D approach derived from the more complex 3D PFSS models of the solar corona (see Luhmann et al. 2002). We find the two typical configurations for the large-scale corona as presented in Fig. 5. Even though this procedure of obtaining a 2D large-scale corona appears very simple, it captures the basic characteristic that there was no large stable polar coronal hole during cycle 23 . In this model, the persistence of a large stable polar coronal hole is coming from the fact that the two flow cells are sinking down over a large range around $60^{\circ}$ to $70^{\circ}$; this in turn is advecting the fields downward and creating the necessary space for the open field lines to spread towards the poles. On the other hand, in cycle 23, the fields did not sink down before being advected up to the pole. Thus the poloidal loops continuously get transported towards the pole where they get squeezed and self-annihilated.

The large-scale coronal dynamics is governed by many other complex processes in the interior as well as photosphere and corona, but the differences in the behavior of the meridional circulation in cycles 22 and 23 produce some trend in the evolving large-scale fields which in turn can shed some light about what to expect for the large-scale coronal behavior.

Acknowledgements I thank Peter Gilman for reviewing the entire manuscript and for his helpful comments. This work is partially supported by NASA grant NNX08AQ34G. I also acknowledge the support from ISSI Bern, for my participation in the ISSI workshop on Cosmic Rays in the Heliosphere II, and the opportunity for the interactions with scientists worldwide working in that area of research. The National Center for Atmospheric Research is sponsored by the National Science Foundation.

Open Access This article is distributed under the terms of the Creative Commons Attribution Noncommercial License which permits any noncommercial use, distribution, and reproduction in any medium, provided the original author(s) and source are credited.

\section{References}

V. Abramenko, V. Yurchyshyn, J. Linker, Z. Mikić, J. Luhmann, C.O. Lee, Low-latitude coronal holes at the minimum of the 23rd solar cycle. Astrophys. J. 712, 813 (2009)

H.W. Babcock, H.D. Babcock, The Sun's magnetic field, 1952-1954. Astrophys. J. 121, 349 (1955)

S. Basu, H.M. Antia, Changes in solar dynamics from 1995 to 2002. Astrophys. J. 585, 553 (2003)

A. Bonanno, D. Elstner, G. Rüdiger, G. Belvedere, Parity properties of an advection-dominated solar alpha ${ }^{2}$ Omega-dynamo. Astron. Astrophys. 390, 673 (2002)

M. Dikpati, Global MHD theory of tachocline and the current status of large-scale solar dynamo. ESA SP 559, 233 (2004)

M. Dikpati, A.R. Choudhuri, The evolution of the Sun's poloidal field. Astron. Astrophys. 291, 975 (1994)

M. Dikpati, P. Charbonneau, A Babcock-Leighton flux transport dynamo with solar-like differential rotation. Astrophys. J. 518, 508 (1999)

M. Dikpati, M. Rempel, P.A. Gilman, K.B. MacGregor, Comments on "Full-sphere simulations of circulationdominated solar dynamo: exploring the parity issue". Astron. Astrophys. 437, 699 (2005)

M. Dikpati, G. de Toma, P.A. Gilman, Predicting the strength of solar cycle 24 using a flux-transport dynamobased tool. Geophys. Res. Lett. 33, L05102 (2006)

M. Dikpati, P.A. Gilman, R.P. Kane, Length of a minimum as predictor of next solar cycle's strength. Geophys. Res. Lett. 37, L06104 (2010a)

M. Dikpati, P.A. Gilman, G. De Toma, R.K. Ulrich, Impact of changes in the Sun's conveyor-belt on recent solar cycles. Geophys. Res. Lett. 37, L14107 (2010b)

V. Domingo, I. Ermolli, P. Fox, C. Fröhlich, M. Haberreiter, N. Krivova, G. Kopp, W. Schmutz, S.K. Solanki, H.C. Spruit, Y. Unruh, A. Vögler, Solar surface magnetism and irradiance on time scales from days to the 11-year cycle. Space Sci. Rev. 145, 337 (2009)

P. Dunzlaff, B. Heber, A. Kopp, O. Rother, R. Müller-Mellin, A. Klassen, R. Gómez-Herrero, R. WimmerSchweingruber, Observations of recurrent cosmic ray decreases during solar cycles. Ann. Geophys. 26, 3127 (2008)

C. Fröhlich, Evidence of a long-term trend in total solar irradiance. Astron. Astrophys. 501, L27 (2009)

G.A. Guerrero, J.D. Muñoz, Kinematic solar dynamo models with a deep meridional flow. Mon. Not. R. Astron. Soc. 350, 317 (2004) 
D.A. Haber, B.W. Hindman, J. Toomre, R.S. Bogart, R.M. Larsen, F. Hill, Evolving submerged meridional circulation cells within the upper convection zone revealed by ring-diagram analysis. Astrophys. J. 570, 855 (2002)

D.H. Hathaway, Does the current minimum validate (or invalidate) cycle prediction methods? SOHO-23 Conference Proceedings on Understanding a Peculiar Solar Minimum, ed. by S.R. Cranmer, J. Todd Hoeksema, J.L. Kohl. San Francisco: ASPC, 428, 307 (2010)

H. Hotta, T. Yokoyama, Solar parity issue with flux-transport dynamo. Astrophys. J. 714, L308 (2010)

R. Howe, J. Christensen-Dalsgaard, F. Hill, R. Komm, J. Schou, M.J. Thompson, A note on the torsional oscillation at solar minimum. Astrophys. J. 701, L87 (2009)

J. Jiang, R.H. Cameron, D. Schmitt, M. Schüssler, Can surface flux transport account for the weak polar field in cycle 23? Space Sci. Rev. doi:10.1007/s11214-011-9783-y (2011, this issue)

L. Jouve, A.S. Brun, On the role of meridional flows in flux transport dynamo models. Astron. Astrophys. 474, 239 (2007)

L. Jouve, A.S. Brun, R. Arlt, A. Brandenburg, M. Dikpati, A. Bonanno, P.J. Käpylä, D. Moss, M. Rempel, P. Gilman, M.J. Korpi, A.G. Kosovichev, A solar mean field dynamo benchmark. Astron. Astrophys. 483, 949 (2008)

M.S. Kirk, W.D. Pesnell, C.A. Young, S.A. Hess Webber, Automated detection of EUV polar coronal holes during solar cycle 23. Sol. Phys. 257, 99 (2009)

M. Küker, G. Rüdiger, M. Schultz, Circulation-dominated solar shell dynamo models with positive alphaeffect. Astron. Astrophys. 374, 301 (2001)

R.B. Leighton, A magneto-kinematic model of the solar cycle. Astrophys. J. 166, 1 (1969)

J.G. Luhmann, Y. Li, C.N. Arge, P.R. Gazis, R. Ulrich, Solar cycle changes in coronal holes and space weather cycles. J. Geophys. Res.-Atmos. 107, 1154 (2002)

N.R. Sheeley Jr., What's so peculiar about the cycle 23/24 solar minimum? ASPC 428, 3 (2010)

S.C. Solomon, T.N. Woods, L.V. Didkovsky, J.T. Emmert, L. Qian, Anomalously low solar extremeultraviolet irradiance and thermospheric density during solar minimum. Geophys. Res. Lett. 37, L16103 (2010)

S.C. Tripathy, K. Jain, F. Hill, J.W. Leibacher, Unusual trends in solar P-mode frequencies during the current extended minimum. Astrophys. J. 711, L84 (2010)

R.K. Ulrich, The controversial sun, in Inside the stars; Proceedings of the 137th IAU Colloquium, ed. by W.W. Weiss, A. Baglin (1993), p. 25

R.K. Ulrich, Solar meridional circulation measured by Doppler shifts of the Fe I line at $\lambda 5250 \AA$ A . Astrophys. J. 725, 658 (2010)

R.K. Ulrich, J.E. Boyden, The solar surface toroidal magnetic field. Astrophys. J. 620, L123 (2005) 